$12-17-2019$

\title{
Individual Based Modeling And Analysis Of Pathogen Levels In Poultry Chilling Process
}

Zachary McCarthy

Cleveland State University, zjm@yorku.ca

Ben Smith

Public Health Agency of Canada

Aamir Fazil

Public Health Agency of Canada

Jianhong Wu

York University

Shawn D. Ryan

Cleveland State University, s.d.ryan@csuohio.edu

Follow this and additional works at: https://engagedscholarship.csuohio.edu/scimath_facpub

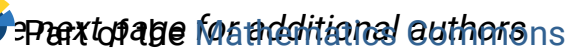

How does access to this work benefit you? Let us know!

Publisher's Statement

https://doi.org/10.1016/j.mbs.2017.10.010

\section{Repository Citation}

McCarthy, Zachary; Smith, Ben; Fazil, Aamir; Wu, Jianhong; Ryan, Shawn D.; and Munther, Daniel, "Individual Based Modeling And Analysis Of Pathogen Levels In Poultry Chilling Process" (2019). Mathematics Faculty Publications. 301.

https://engagedscholarship.csuohio.edu/scimath_facpub/301

This Article is brought to you for free and open access by the Mathematics and Statistics Department at EngagedScholarship@CSU. It has been accepted for inclusion in Mathematics Faculty Publications by an authorized administrator of EngagedScholarship@CSU. For more information, please contact library.es@csuohio.edu. 


\section{Authors}

Zachary McCarthy, Ben Smith, Aamir Fazil, Jianhong Wu, Shawn D. Ryan, and Daniel Munther 


\title{
Individual based modeling and analysis of pathogen levels in poultry chilling process
}

\author{
Zachary McCarthy ${ }^{\mathrm{a}}$, Ben Smith ${ }^{\mathrm{c}}$, Aamir Fazil ${ }^{\mathrm{c}}$, Jianhong Wu ${ }^{\mathrm{b}}$, Shawn D. Ryan ${ }^{\mathrm{a}}$, Daniel Munther ${ }^{:, a}$ \\ a Department of Mathematics, Cleveland State University, Cleveland, OH, USA \\ b Laboratory for Industrial and Applied Mathematics, York University, Toronto, ON, Canada \\ c Public Health Risk Sciences Division, National Microbiology Laboratory, Public Health Agency of Canada, Guelph, ON, Canada
}

\section{Introduction}

Given the increase in poultry consumption and the globalization of the food supply chain, food-borne pathogens in general and, Campylobacter spp. in particular, have become leading causes of human enteric infections worldwide [1,2]. In order to alleviate both the public health and socio-economic burden associated to such illnesses, researchers have conducted numerous studies, attempting to enumerate pathogen prevalence and concentration at various processing stages (see for instance [3-8] and references therein). While the results from these studies are valuable in their own right, these findings have to be integrated to inform quantitative microbial risk assessment (QMRA) models as a management tool for food safety control [1,2]. Such "stochastic risk models are robust tools that can be used for evidence-informed decision-making, and are uniquely suited to manage a wide breadth of data and interpret the variability and uncertainty inherent of microbial populations" [1]. However, pathogen transfer probabilities at key steps during poultry processing may be unknown, difficult to extract from experimental studies, or loosely estimated, limiting the confidence in predictive results. In particular, the underlying mechanisms of cross-contamination during immersion chilling are still poorly understood, and furthermore, studies evaluating the efficacy of intervention strategies during chilling have presented inconsistent and even contradictory results [9]. Accordingly, as chilling is one of the last stages of processing, designed to arrest microbial growth on carcasses by lowering their temperature, more insight is needed to elucidate cross-contamination mechanisms as well as effective strategies for pathogen control.

To begin addressing these issues, Munther et al. [10] developed a preliminary mechanistic model quantifying the average cross-contamination of poultry carcasses in the chill tank with generic $E$. coli, describing the chiller process of a typical modernized Canadian poultry inspection program plant (high speed). An important attribute of the model is that it provides quantifiable links between processing control parameters and microbial levels, simplifying the complexity of these relationships to provide guidance for developing and parameterizing models that are able to predict both pathogen prevalence and carcass contamination levels. In this work, we expand this chiller model into a format amenable to directly inform existing QMRA models for Campylobacter in poultry [1]. In particular, the newly developled IBM can incorporate stochastic inputs in terms of pre-chiller (i.e. before entering the chill tank) contamination levels as well as pre-chiller organic load and can track pathogen levels on individual birds throughout the chilling process. 
The paper is organized as follows: in Section 2 we discuss the background and modeling formulation. In Section 3 we provide detailed parameter estimation specific to Campylobacter contamination and in Section 4 we present our numerical approach. In Section 5, we discuss the results of our model simulations, comparing both deterministic and stochastic predictions and discussing the implications of free chlorine (FC) control. Furthermore, we provide model validation against recent experiments, followed by a sensitivity analysis for relevant model parameters. Finally, in Section 6 we summarize our results in the context of QMRA implementation and highlight important future directions of study.

\section{Background and modified chiller model}

Canada has a variety of poultry processing operations, ranging from smaller traditional type processing to state of the art, high speed facilities. In this paper, we suppose that the processing framework involves a poultry processing establishment (high speed) that follows guidelines under the Canadian Food Inspection Agency (CFIA) approved Modernized Poultry Inspection Program (MPIP). This basis leads to the following assumptions that guide our model design: (i) the mass of a carcass is $2 \mathrm{~kg}$; (ii) the processing speed is 180 carcasses/min; (iii) the average dwell time of carcasses in the chiller tank is $45 \mathrm{~min}$; (iv) chiller water is not recycled, rather the set up involves fresh water intake at the beginning of the chiller tank, with overflow at the end; (v) a maximum of $50 \mathrm{ppm}(\mathrm{mg} / \mathrm{L})$ of free chlorine is added (if any) at the beginning of the chiller tank, mixing with incoming fresh water; and (vi) organic material and bacteria do not attach to chiller tank surfaces [10].

Similar to the setup in [10], our model of the chilling process includes two general types of mechanisms: (I) industrial specifications related to high speed poultry slaughter establishments in Canada and (II) bacteria transfer/inactivation and chiller water chemistry dynamics. For the sake of clarity, mechanisms described by type (I) parameters are what links our model to particular Canadian processing specifications. Type (II) mechanisms are expected for general largescale chilling operations and are not limited to Canada. Therefore, data used to inform type (II) mechanisms need not be limited to Canada.

\subsection{Carcass dynamics and organic material in the tank}

Let $p_{i}$ represent carcass $i$, and let $N$ be the total number of processed carcasses. Based on a 45 min dwell time, we assume carcass $p_{1}$ enters the tank at time $t_{1}$ and leaves the tank at time $\hat{t}_{i}=t_{i}+45$.

As the chickens enter and move through the chiller tank, they release high amounts of organic material (in the form of blood, fat, protein, etc.) into the water. Such material is important because it alters chiller water chemistry as well as microbial counts [11]. We represent the amount of organic material on carcass $p_{t}$ at time $t>0$ by $J_{p}$ ( $(\mathrm{kg})$. In particular, we assume that the incoming level of organic material on carcasses is proportional to the mass of the carcass. Therefore before entering the tank, we have $J_{p_{i}}(0)=J_{p_{i 0}}=q m_{c}$, where $q \in(0,1)$ and $m_{c}$ $(\mathrm{kg})$ is the mass of the carcass. Furthermore, we assume that the shed rate of the organic material from each carcass into the chiller water is proportional to the current organic material amount on the carcass. This leads to the following dynamics for $J_{p_{i}}$ :

$J_{p_{i}}^{\prime}= \begin{cases}-\gamma J_{p_{i}} & t_{\mathrm{i}} \leq t<\hat{t}_{i} \\ 0 & \text { otherwise }\end{cases}$

Here the prime is the derivative with respect to time. Notice that there is only shed from carcass $p_{1}$ for $t \in\left[t_{i}, \hat{t}_{i}\right)$, i.e. when $p_{1}$ is in the chiller tank. While in reality the amount of organic material shed from individual carcasses may be independent of one another, we assume $\gamma>0(1 / \mathrm{min})$ is the average shed rate for each carcass.

To describe the dynamics of the organic material in the tank, we let
$J_{W}$ represent the current amount of organic material in the chiller water. Assuming that this material is added to the water via carcass shedding and leaves the tank via water outflow, we have:

$J_{W}^{\prime}=\sum_{i=1}^{N} \gamma_{i} J_{p_{i}}-\frac{g}{T_{V} 10^{-3}} J_{W}$

where

$\gamma_{i}= \begin{cases}\gamma & t_{i} \leq 1<\hat{\ell}_{t} \\ 0 & \text { utherwise }\end{cases}$

Notice the first term in Eq. (2) is governed by $\gamma_{i}$ from (3), which allows shed only from carcasses that are actually in the tank. The second term in Eq. (2) quantifies the rate at which organic materials leave the tank due to outflow. We assume the tank volume $T_{V}(\mathrm{~mL})$ is constant in time, so inflow = outflow. Also, we let $R(\mathrm{~kg} / \mathrm{min})$ be the incoming rate of carcasses. Assuming that $l$ liters of fresh water are added to the tank per carcass and each carcass on average weighs $m_{c} \mathrm{~kg}, \mathrm{~g}=R(\mathrm{~kg} / \mathrm{min}) \times(1$ carcass $\left./ m_{\ell} \mathrm{kg}\right) \times l(\mathrm{~L}$ freshwater $/$ carcass $)=R l / m_{c},(\mathrm{l} / \mathrm{min})$ is the addition rate of fresh water per carcass.

\subsection{Microbial dynamics in the tank}

We assume the following three aspects determine the bacterial level, $v_{p_{i}}$ (CFU), on carcass $p_{1}$ during chilling: (i) bacteria shed into chiller water from $p_{i}$; (ii) bacteria on $p_{t}$ are inactivated via chlorine; and (iii) bacteria attach to the surface of $p_{i}$ from contact between the carcass and bacteria in the chiller water. Following (i)-(iii), the equation for $v_{p}$ is

$v_{p_{i}}^{\prime}= \begin{cases}m_{i} T_{V} \hat{p} W-b v_{p_{1}}-\alpha k_{w} \cdot v_{p_{i}} C & \hat{b}_{i} \leq 1<\hat{\imath}_{i} \\ 0 & \text { otherwise }\end{cases}$

Note that carcass $p_{i}$ enters the chiller tank with bacteria load $\sigma_{i}=v_{P_{i}}(0)$, and as it moves through the chiller tank, we suppose that continued microbial shedding occurs at a rate $b v_{p}$, where $b(1 / \mathrm{min})$ is the shedding parameter (i.e., the shedding rate is proportional to the current contamination level on the poultry). In addition, let $W(\mathrm{CFU} / \mathrm{mL})$ be the microbial concentration in the chiller water at time $t$, then we assume bacterial attachment occurs at a rate $m_{s} T_{V} \beta W$, where $\beta(1 /(\mathrm{kg} \min ))$ is the binding parameter.

In addition to shedding/binding, we consider the inactivation of microbes on carcass surfaces via free chlorine (FC) contact during the chiller process. While the effective contact of FC with carcass surfaces during immersion chilling is complex due to surface morphology affecting various degrees of microbial attachment [12], we take a simplified approach (similar to the approach in [10]). For instance, multiple studies provide clear quantification of inactivation rates of microbes in solution via FC; see $[13,14]$ and references therein. If we let $k_{w}>0$ be the inactivation rate of microbes in the chiller water, then we argue that the inactivation rate via $\mathrm{FC}$ of microbes on carcass surfaces can be written as $\alpha k_{w}$, where $\alpha \in(0,1)$ (see [10] for more details). Since carcass surfaces are irregular and this is an important factor in determining contamination levels [3], FC contact with microbes attached to carcass surfaces should be significantly less than FC contact with microbes in the chiller water. Combining these ideas, the decrease of the microbial load on carcasses is given by $\alpha k_{w}, v_{p} C$. where we assume that this decrease is proportional to the product of the current microbial load on $p_{i}$ and the FC concentration $\mathrm{C}(\mathrm{mg} / \mathrm{L})$.

Next, we build a dynamic equation for the concentration of pathogens in the chiller water, $W$. We assume that chiller water is not filtered or recycled, bacteria do not multiply in the water because of the low temperature, $\leq 4{ }^{\circ} \mathrm{C}$ as per CFIA regulations, and bacterial survival in the water is expected $[15,16]$. Note that results from [17] indicate that the average temperature just under the skin of a $2 \mathrm{~kg}$ carcass, subject to water temperatures $\leq 4^{\circ} \mathrm{C}$, takes 5 to $10 \mathrm{~min}$ on average to cool from its prechill temperature $\left(33-40{ }^{\circ} \mathrm{C}\right)$ to $4{ }^{\circ} \mathrm{C}$. While this indicates the possibility of bacteria growth during this phase, results from [4], 
suggest that this growth is most likely not significant. Thus, $W$ depends on the following: (B1) bacteria shed into the water, (B2) bacteria in water attaching to poultry and organic material, (B3) bacteria inactivated by FC and (B4) bacteria flowing out of the tank with water overflow. While there may be concentration differences along the length of the tank, for simplicity we assume complete mixing for the dynamics in (B1)-(B4) and formulate the equation for $W$ as follows:

$\boldsymbol{W}^{\prime \prime}=\sum_{i=1}^{N} b_{i} v_{P_{i}}-W\left(\sum_{i=1}^{N} \beta_{i} m_{e}\right)-\beta J_{W} W-k_{w} C W-\frac{g}{T_{V} 10^{-3}} W$

where

$b_{i}= \begin{cases}b & l_{i} \leq t<\hat{\ell}_{i} \\ 0 & \text { otherwise }\end{cases}$

and

$\beta_{i}= \begin{cases}\beta & t_{1} \leq t<\hat{t}_{i} \\ 0 & \text { otherwise }\end{cases}$

Note that $b_{1}$ and $\beta$, dictate the microbial shed and binding parameters, ensuring that only carcasses in the tank are exposed to these dynamics. Also, consider that injured bacteria cells might also be represented in this model, given the assumption that they shed from/adhere to carcasses at the same rates as other intact/viable cells. However, in the current model, we do not distinguish between injured and viable cells.

\subsection{Chlorine kinetics in the tank}

The equation governing the $\mathrm{FC}$ concentration in the $\operatorname{tank} \mathrm{C}(\mathrm{mg} / \mathrm{L})$ is

$C^{\prime}=\frac{c_{i} g}{10^{-3} T_{V}}-h_{w} C J_{W}-C\left(\sum_{i=1}^{N} h_{i} J_{P_{i}}\right)-\frac{g}{10^{-3} T_{V}} C$

where

$h_{i}= \begin{cases}h_{w} & t_{i} \leq t<\hat{t}_{i} \\ 0 & \text { otherwise }\end{cases}$

and where $c_{1}(\mathrm{mg} / \mathrm{L})$ is the FC concentration of the input water, $g$ is as above, and so $c_{1} g /\left(10^{-3} T_{V}\right)(\mathrm{mg} /(\mathrm{L} \mathrm{min}))$ measures the rate of increase of $\mathrm{FC}$ in the water. Also, $h_{w}(1 /(\mathrm{kg} \mathrm{min}))$ is the rate at which the organic material in the tank decreases the FC through chemical binding. Notice that $h_{i}$ is equal to $h_{w}$, when $p_{i}$ is in the tank and is 0 otherwise. For the terms involving $h_{w}$ and $h_{t}$ we assume that the decrease in FC concentration at time $t$ is proportional to the product of the respective interacting "species", and therefore these terms represent a type of second order rate constant. Finally, $\mathrm{gC} /\left(10^{-3} T_{V}\right)$ quantifies the loss of $\mathrm{FC}$ due to outflow of water from the tank.

\subsection{Complete model}

Combining Equs. (1) to (9), the complete IBM becomes:

$$
\begin{aligned}
& J_{P_{i}}^{x}=\left\{\begin{array}{ll}
-\gamma J_{P_{i}} & t_{i} \leq t<\hat{t}_{i} \\
0 & \text { otherwise }
\end{array} \text { for } i=1, \cdots, N\right. \\
& J_{W}^{\prime}=\sum_{i=1}^{N} \gamma_{i} J_{D_{i}}-\frac{\mathrm{g}}{T_{b^{\prime}} \cdot 10^{-3}} J_{W} \\
& v_{p_{i}}^{\prime}=\left\{\begin{array}{ll}
m_{i} T_{V} \beta W-b v_{p_{i}}-a k_{w^{\prime}} v_{p_{i}} C & t_{i} \leq t<\hat{t}_{i} \\
0 & \text { otherwise }
\end{array} \text { for } l=1, \cdots, N\right. \\
& W^{\prime}=\sum_{i=1}^{N} b_{i} v_{p_{i}}-W\left(\sum_{i=1}^{N} \beta_{i} m_{\epsilon}\right)-\beta J_{W} W-k_{H} C W-\frac{g}{T_{V} 10^{-3}} W \\
& C^{\prime}=\frac{c_{1} g}{10^{-3} T_{V}}-h_{W^{\prime}} C J_{\mathcal{H}}-C\left(\sum_{i=1}^{N} h_{i} J_{p_{i}}\right)-\frac{g}{10^{-3} T_{V^{\prime}}} C .
\end{aligned}
$$

with initial conditions: $J_{p_{i}}(0)=J_{p_{i 0}}=q m_{i} \cdot J_{W}(0)=J_{W 0}, v_{p_{i}}(0)=\sigma_{i}$, $W(0)=W_{0}$, and $C(0)=C_{0}$. For the explicit equations for $\gamma_{1}, b_{\dot{v}} \beta_{1}$ and $h_{1}$, please refer to Eqs. (3), (6), (7), and (9), respectively. Model variables and parameters are also summarized in Table 1.

\subsection{Well-posedness}

To show that model (10) is well-posed, we first consider the linear system

$\frac{d x}{d t}=A(t) x+b(t)$

where $x(t)$ and $b(t)$ are $n$-dimensional vector valued functions and $A(t)$ is a $n \times n$ matrix. We can use the following theorem from ([18], p. 5): Let all the elements of the matrix $A(t)$ and the vector-valued function $b(t)$ be summable (i.e. Lebesque integrable) on each segment contained in the interval $(a, b)$. Then for $t_{0} \in(a, b)$, the solution of the system (11) with arbitrary initial datax $\left(t_{0}\right)=x_{0}$ exists on the whole interval $(a, b)$ and is unique.

To show the existence and uniqueness of solutions for (10), we proceed by repeatedly applying this theorem. Notice the right hand side of (10) has only finitely many discontinuities in the variable $t$, determined by indicator functions scaled by non-zero constants that are "on" when the ith carcass $\left(p_{i}\right)$ is in the tank (i.e. from time $\left.\mid t_{i}, \hat{t}_{i}\right]$ ) and are "off" otherwise. To apply the above theorem, let $(a, b)=\left(-1, T^{*}+2\right)$, where we say $t_{1}=0$ (i.e. the time when $p_{1}$ enters the tank) and let $\hat{t}_{N}=T^{*}$ (i.e. the time when the last carcass, $p_{N}$, leaves the tank). Then let $\left[a_{1}, b_{1}\right]=\left[0, T^{*}+1\right]$. Applying the above theorem, the equations for $J_{m}^{\prime}$ and $J_{W^{\prime}}^{\prime}$ in (10) have unique continuous solutions on $\left[0, T^{*}+1\right]$. Thus, the equation for $C^{\prime}$ also has a unique continuous solution on $\left[0, T^{*}+1\right]$. Next, plugging in the solutions for $J_{p ;}, C$, and $J_{W}$ into the equations for $v_{p}^{\prime}$ and $W^{\prime}$, we again can apply the theorem, and conclude that (10) has a unique continuous solution on $\left[0, T^{*}+1\right]$. Furthermore, notice that for time $t \geq T^{*}, J_{P_{i}}$ and $v_{p \text {. }}$ are constant for all $i$ (as all carcasses have exited the chill tank). One can easily show that $J_{W} \rightarrow 0$, $C \rightarrow$ positive constant and $W \rightarrow 0$ as $t \rightarrow \infty$. Thus, for $t \geq 0,(10)$ has continuous, uniformly bounded solutions.

In terms of positivity of solutions of (10), it is clear by inspection that $J_{p,}$ (for all $i$ ), $J_{W}$ and $C$ all remain non-negative for all $t \geq 0$. It remains to show that $W$ and $v_{k}$ are non-negative for all $t \geq 0$. Note that

Table 1

Model variables and parameters used for application to Campylobacter contamination during chilling.

\begin{tabular}{lll}
\hline Symbol & Initial Value/Values/Units & Description, Reference \\
\hline$v_{v_{i}}$ & $\sigma_{i} \in U\left[1 \times 10^{4}, 1 \times 10^{4}{ }^{6}\right] \mathrm{CFU}$ & Campy. count on $p_{i},[7]$ \\
$J_{P_{i}}$ & $J_{P U} \in U[0,0.02] \mathrm{kg}$ & Organic material on $p_{i}$ \\
$C$ & $C_{0}=0, c_{1}=\{0,25,50\} \mathrm{mg} / \mathrm{L}$ & FC concentration in water/ FC input, \\
& & {$[19]$} \\
$W$ & $W_{0}=0 \mathrm{CFU} / \mathrm{ml}$ & Campy. concentration in water \\
$J_{W}$ & $J_{W, 0}=0 \mathrm{~kg}$ & Organic material in water \\
$T$ & $480 \mathrm{~min}$ & Total simulation time \\
$N$ & 80,000 & Chickens processed \\
$\gamma$ & $0.05 \mathrm{~min}^{-1}$ & Shed rate of organics from $p_{i}$ \\
$m_{C}$ & $2 \mathrm{~kg}$ & mass of $p_{i}$, [P] \\
$\beta$ & $0.001(\mathrm{~kg} \mathrm{~min})^{-1}$ & Campy. attachment rate \\
$b$ & $0.077 \mathrm{~min}^{-1}$ & Campy. shed rate, [4] \\
$a$ & 0.000001 & Fraction FC kill rate on $p_{i},[4,6,7]$ \\
$k_{w}$ & $143 \mathrm{~L}\left(\mathrm{mg} \mathrm{min}^{-1}\right.$ & FC kill rate of Campy. in water, [20] \\
$T_{V}$ & $5 \times 10^{7} \mathrm{~mL}^{-1}$ & Chiller tank volume, [22] \\
$g$ & $306 \mathrm{~L} \mathrm{~min}{ }^{-1}$ & Input water rate, [P] \\
$h_{w}$ & $0.0017(\mathrm{~kg} \mathrm{~min})^{-1}$ & FC oxidation rate via organics, [21] \\
\hline
\end{tabular}

Note: $p_{l}$ represents carcass $i$ and $U$ indicates a uniform distribution over the respected range. Parameters with reference $[\mathrm{P}]$ correspond to information obtained from personal communication from CFIA officers. Please refer to Section 3 for more details on the respective parameter values/ranges indicated above. 
$W(0)=0$ and $v_{p_{1}}(0)=\sigma_{1}>0$. Thus, at $t=0 . W^{\prime}=b_{1} v_{p_{1}}>0$. Because $W$ is continuous, it either stays positive for all $t>0$, or there exists a time $\tau_{1}>0$, such that $W\left(\tau_{1}\right)=0$ and $W(t)>0$ for $t \in\left(0, \tau_{1}\right)$. The only case that needs discussion is when, $\tau_{1} \in\left[\hat{\iota}_{k}, \hat{t}_{k+1}\right)$ for some $k \in\{1, \cdots, N-1\}$, where $\hat{t}_{i}$ is the time when $p_{1}$ leaves the tank. In this case, at time $\tau_{1}$, chickens $k+1, \cdots, j$ are in the tank. So,

$W^{\prime \prime}\left(\pi_{1}\right)=\sum_{i=k+1}^{L} b_{i} v_{P_{i}}$

Notice for carcasses $i=k+1, \cdots, j$, for $t \in\left[t_{i}, \tau_{1}\right]$,

$v_{p_{i}}^{\prime}=m_{\varepsilon} \beta T_{V} W-b v_{p_{i}}-\alpha k_{w} v_{p_{i}} C$

$\geq-b v_{p_{i}}-\alpha k_{w} v_{p_{i}} C$, since $W \geq 0$, on $\left[0, \tau_{1}\right]$.

Therefore,

$v_{p_{i}} \geq \sigma_{i} e^{\hat{l}_{i}^{\theta_{1}}-b-\alpha k_{w} C(s) d s}>0$, since $\sigma_{i}>0$.

Going back to $W$, this means that

$W^{\prime}\left(\tau_{1}\right)=\sum_{i=k+1}^{j} b_{i} v_{p_{i}}>0$

Thus, $W$ cannot become negative while any carcasses are in the tank (i.e. for $t \in\left[0, T^{*}\right]$ ). Furthermore, when all carcasses have exited the tank, i.e. for $t>T^{\circ}$, it is clear that $W \geq 0$. Considering the formula for $v_{p_{i}}^{\prime}$ in (10), this implies that $v_{p_{i}} \geq 0$ for all $t \geq 0$ as well.

Thus, we have shown that solutions (with non-negative and not identically zero initial conditions) of 10 are biologically relevant for the chilling process as they are non-negative and bounded for all forward time.

\section{Parameter estimation for Campylobacter contamination}

\subsection{Campylobacter load on incoming carcasses}

$\sigma_{i}$ : For our simulation results (see Section 5), following the experimental setup in [7], we assume that the incoming Campylobacter load on carcasses, $\sigma_{i}$, ranges over $\left[10^{4}, 10^{4.6}\right] \mathrm{CFU}$. Note that from [4], the average Campylobacter load on pre-chill carcasses (i.e. carcasses about to enter the chill tank) was roughly $10^{4.6} \mathrm{CFU} /$ carcass. However, prechill data in [5] indicate possible loads on the order of $10^{6} \mathrm{CFU} /$ carcass or higher.

\subsection{FC input, fresh water addition and overflow}

We assume that $c_{1}$ ranges from 0 to $50 \mathrm{ppm}$ as per USDA law [19], a maximum of $50 \mathrm{ppm}(\mathrm{mg} / \mathrm{L})$ of free chlorine can be added to the chiller tank (with freshwater input). Since on average $1.7 \mathrm{~L}$ of water is required per carcass and we assume that the rate of carcass processing is about 180 carcasses per minute, then the water use rate, $\mathrm{g}$, is about $306 \mathrm{~L}$ / $\min$.

\subsection{Shed rate of Campylobacter from carcasses to chiller water}

$b$ : Using pre and post-chill Campylobacter data from [4] we estimate the shed parameter $b$ to be about $0.077\left(\mathrm{~min}^{-1}\right)$ on average (similar to generic $E$. coli). See [10] for details of similar type calculations.

\subsection{Shed rate of organic material from carcasses to chiller water}

$\gamma$ : The parameter $\gamma$ dictates the shed of organic material from a carcass into the chiller water. We estimate $\gamma \approx 0.05\left(\mathrm{~min}^{-1}\right)$. This estimate is reasonable as it translates to carcasses leaving the chill tank with less than $1 \%$ of pre-chill organic material remaining.

\section{5. $F C$ inactivation kinetics}

$k_{w}$ and $\alpha k_{w}$ : Using data from [20] and the model form $W^{\prime}=-k_{v} C W$. we estimate $k_{w}$, the rate at which Campylobacter is inactivated by FC in water. Notice that experiments in [20] were conducted at $4^{\circ} \mathrm{C}$ which closely resembles the chiller water temperature. Averaging across inactivation rates for two different Campylobacter strains, $\mathrm{pH}$ values at 6 and 8 , and an experimental time interval of $30 \mathrm{~s}$, we estimate $k_{w^{\prime}}=143$ $\left(\min ^{-1}\right)$.

In order to determine the kill rate of Campylobacter on carcass surfaces, we use the model form $v_{p}^{\prime}=-\alpha k_{w} v_{p} C$ to estimate the inactivation rate, $\alpha k_{w}$, from three studies. From the study in [6], which examined both 0 -hour and 8 -hour chiller water, $\alpha k_{w^{\prime}} \approx 10^{-4}\left(\mathrm{~min}^{-1}\right)$, regardless of chiller water age. Estimates using data from [4] and [7], indicate the same order of magnitude for $\alpha k_{w}$. Therefore, because $k_{w}$ is on the order of $10^{2}$ and $\alpha k_{w} \approx 10^{-4}$, we set $\alpha=10^{-6}$.

\subsection{Binding rate of Campylobacter to poultry in tank}

$\beta$ : Dictating the binding rate of Campylobacter in the chiller water to carcasses in the chill tank, we estimate $\beta \approx 0.001$. While we have no direct data for this we refer to [10] for a detailed discussion of this parameter and methods of estimation.

\subsection{Organic load on incoming carcasses}

$J_{p}(0)$ : We quantify the initial amount of organic material on carcass $p_{i}$ by $J_{p_{i}}(0)=q m_{c}$, where $m_{c}=2 \mathrm{~kg}$ (mass of each carcass) and $q \in(0$, 1 ). Due to lack of pertinent data, we make a reasonable estimate that $J_{P:}(0)$ should range from 0 to $0.02 \mathrm{~kg}$. That is, the initial organic load on each carcass ranges from 0 to $1 \%$ of the initial carcass mass of $2 \mathrm{~kg}$.

\subsection{FC consumption kinetics due to organic material in chiller water}

$h_{w}$ : From [21], the chiller water at equilibrium (i.e. later in the processing day) is shown to have a total suspended solids measurement of $\bar{J}_{W}=3500 \mathrm{mg} / \mathrm{L}$ (or $0.35 \%$ ). Assuming, as above, $T_{V}=5 \times 10^{7} \mathrm{~mL}$, we have that for large enough $t, J_{W}(t) \approx J_{W}=J_{W} \frac{3 \times 10^{4} \mathrm{~L}}{10^{6} \mathrm{mg}}=175 \mathrm{~kg}$. Substituting this into the equation of $C^{\prime}$ from model (10) (and ignoring the other dynamics involved), we solve to get

$C(t)=C_{0} e^{-h_{\mathrm{H}^{\prime}} J t}$

Referring to the data in Table 5 of [21], we see that chlorine depletion from organic material has both a "fast" and "slow" kinetic. For our purposes, we consider only the fast kinetic as we have a continuous flow of chlorine and organic material entering the chill tank. From [21], the average of this fast kinetic is $0.29 \mathrm{~min}^{-1}$. Combining this with the rate in (12), gives $h_{\mathrm{w}} J=0.29$. Since $J=175 \mathrm{~kg}$, we use $h_{\mathrm{w}}=0.0017$.

\section{Numerical approach}

In this section, we use model (10) presented above in representative simulations to simulate a full day of chiller processing (e.g. $8 \mathrm{~h}$ ). The main objective is to track the bacteria on each chicken before, during, and after the chilling process. In particular, we are interested in any cross-contamination which could arise throughout the chilling process.

We focus this work and the corresponding simulations in terms of Campylobacter contamination. The main component (in addition to the low temperature) used to control the bacterial content on each chicken is the constant injection of $\mathrm{FC}$ into the tank. In this section, we discuss calculations to describe the dynamics of the bacteria level on each carcass, amount of organic material left on each carcass, free chlorine levels, bacteria level of the water, and organic material in the water. See Table 1 for the representative parameter values used to generate the results in the next section (unless otherwise noted in the corresponding figure). 
Since we simulate a full eight hour day the simulation will need to track approximately 80,000 chickens with their associated bacteria and organic material levels. Combined with the bacteria count in the water, the organic material in the water, and the chlorine levels we track 160,003 quantities. The simulations are setup to account for 8000 chickens occupying the chiller at any one time and the average length of time a chicken spends in the chiller is $45 \mathrm{~min}$. Thus, a chicken should enter (and exit) the chiller every $0.33 \mathrm{~s}$ or 3 chickens/s. Notice that our numerical approach is easily amenable to chiller processing specifics (i.e. other processing speeds, chiller dwell times, etc.).

\subsection{Incorporating stochasticity}

The advantage of using the IBM (10) presented here is that we can track the bacteria dynamics on each chicken over time. In particular, mean-field or averaged models assume all chickens are the same and can only account for what happens on average. In the simulations we can assign a random initial count of bacterial on each chicken, $\sigma_{i}$, and a random amount of initial organic material, $J_{p_{i}}(0)$. As the simulation progresses, the IBM can evolve the distribution in time providing a more accurate expected range for the bacteria count on chickens leaving the tank. This is especially crucial when bacteria levels are near an unacceptable range. Our simulations can predict the percentage chance a given carcass entering the chiller tank at a given time of the work day will leave "unacceptable" or "acceptable" for consumption.

In principle we can sample the bacteria counts from any type of distribution and adjust the simulation accordingly. In this work, we sample from a uniform distribution for $\sigma_{1}$ between $10^{4}-10^{4.6}$ initial bacteria per chicken [7]. Most programming languages have a standard pseudo-random number generator capable of producing a sequence of random numbers drawn from a uniform distribution between zero and one, $\zeta \in U[0,1]$. The range of the distribution can easily be adjusted to any desired values through a simple affine transformation: $\zeta_{\text {campy }}=v_{p, \min }+\left(v_{p, \max }-v_{p, \min }\right) \zeta$. Observe that if $\zeta=1$, then $\zeta_{\text {campy }}=v_{p, \max } \approx 10^{46}$ and if $\zeta=0$, then $\zeta_{\text {ampy }}=v_{p, \max } \approx 10^{4}$. The sequence of numbers generated for each of the bacteria is pseudo-random in that we can assign it a unique identification number so that it can be reproduced at anytime for further analysis. The use of a normal or another distribution is easy to implement as well.

In order to have a fair comparison between the deterministic and the stochastic runs of model (10), we enforce that for a deterministic run, the initial conditions for bacteria levels and organic load in the water are chosen to be the sample average from the stochastic generated values. In the results presented in the next section we will highlight two key points: (i) the additional information obtained from the stochastic simulations and (ii) the justification of our previous averaged model [10] after a sufficient period of time.

\subsection{Effects of a chicken felt long after it leaves the tank}

The unique feature provided by an IBM, in contrast to an averaged model [10], is that we can track the impact of each chicken passing through the chiller tank on all future chickens. For example, as a chicken moves through the tank, it sheds pathogens and organic material from its body, both of which contribute to the increased organic load in the water and bacterial concentrations in the water. These can have a great effect on all following chickens entering the tank (see Figs. 2 and 3 in Section 5).

\subsection{Accounting for time of day}

The simulations presented can also be used to track a particular segment in a given shift. The only changes would involve the initial chlorine levels $\left(C_{0}\right)$, the initial bacteria concentration in the water $\left(W_{0}\right)$, and the initial organic load in the water $\left(J_{w, 0}\right)$. We can also extract particular values of the model variables for a given starting point from

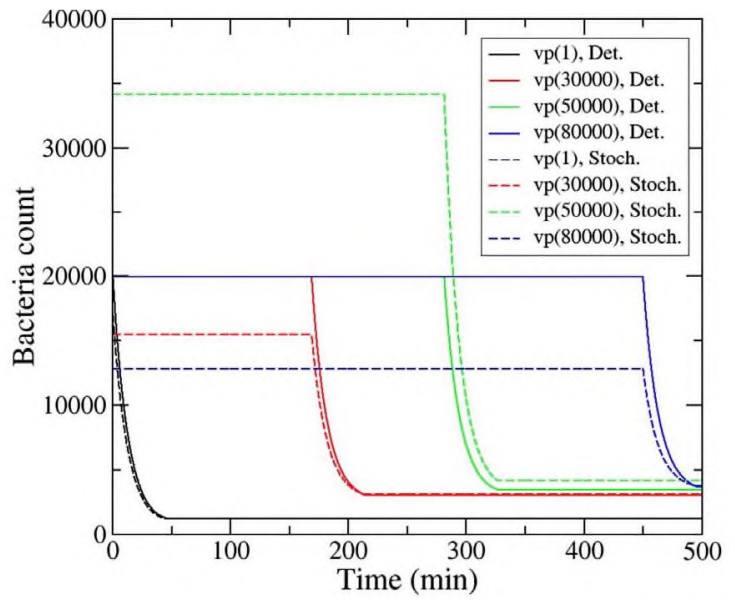

Fig. 1. Dynamics of Campylobacter counts on sample carcasses. Here FC input is $25 \mathrm{mg} / \mathrm{L}$.

our plots for a full day. The other subtle aspect of the numerical simulations involving a particular carcass $p_{t}$ concerns the notion that the carcasses which precede and follow $p_{l}$ during chilling need to be accounted for (unless the time window is near the beginning or end of the day). This is due to the fact that when the ith chicken enters it still has 45 min before it exits while other chickens continue to enter the tank after it. This dynamic must be included for the simulations to accurately capture the chilling process.

\section{Results and discussion for IBM applied to Campylobacter contamination}

Using $25 \mathrm{mg} / \mathrm{L}$ of FC input, we track the Campylobacter levels on three individual birds over time including before entering the tank, inside the tank, and upon leaving the tank, as seen in Fig. 1. Observe that the values can only change while in the tank and the model (10) assumes that the bacteria count is fixed outside the chiller tank. Comparing both deterministic and stochastic results, despite large initial differences in incoming bacterial loads, bacteria counts on carcasses leaving the tank reflect less disparate values. This phenomenon is expected due to the well-mixing assumptions used in the model. Plots with this type of data are easily generated from stochastic runs, but cannot be extracted from a deterministic run where all chickens are assumed to be identical.

In Fig. 2, we present the distribution of Campylobacter levels over time (again with $25 \mathrm{mg} / \mathrm{L} \mathrm{FC} \mathrm{input).} \mathrm{This} \mathrm{clearly} \mathrm{illustrates} \mathrm{the} \mathrm{three}$ phases of the chilling process for each chicken; namely, (i) pre-chilling

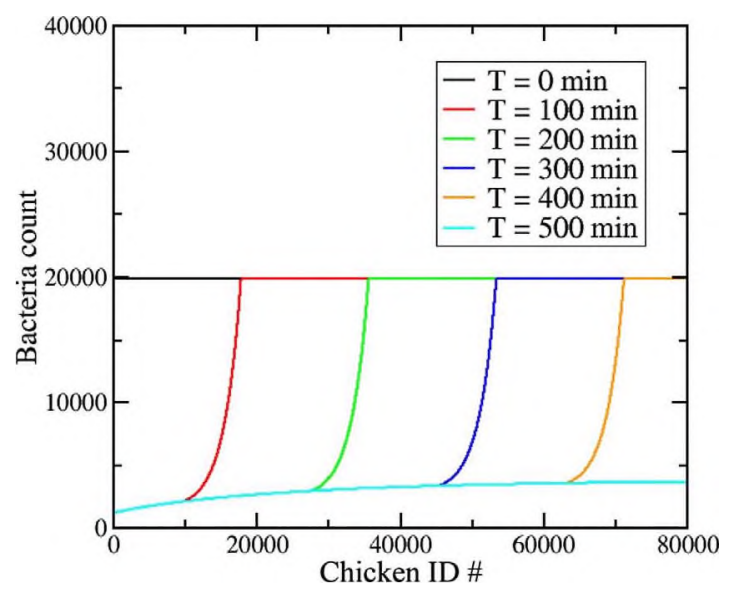

Fig. 2. Distribution of Campylobacter levels over time in the determinisitic simulations. Here FC input is $25 \mathrm{mg} / \mathrm{L}$. 


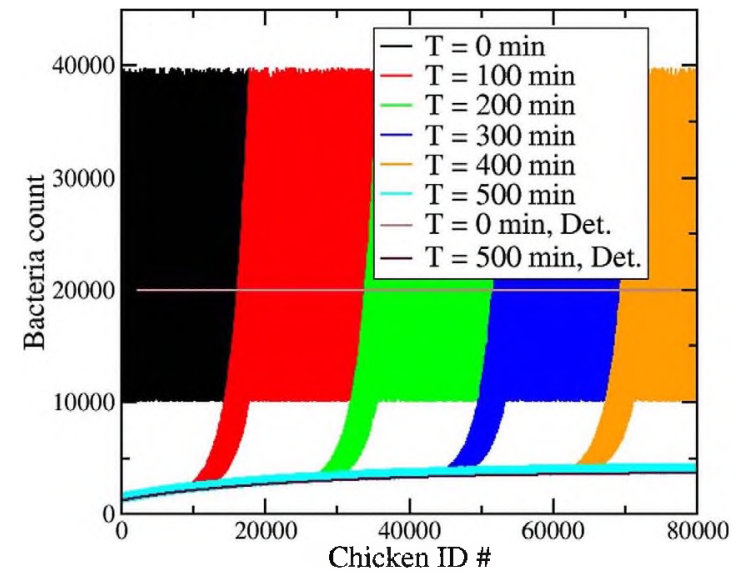

Fig. 3. Distribution of Campylobacter levels over time comparing the deterministic to the stochastic simulations. Note: The y-axis is presented in $\log _{10}$ scale and FC input is $25 \mathrm{mg}$ / L.

represented by the horizontal line capturing the initial condition in the deterministic case, (ii) inside the tank, which is marked by a rapid change in the bacteria level showing a decrease (effective killing via $\mathrm{FC} /$ shedding), (iii) the bacterial load remains at its exit level for the rest of the simulation time.

We can see in both simulations (deterministic in Fig. 2 and stochastic in Fig. 3) that cross-contamination is more likely toward the end of the day. This can be explained by the buildup of the organic load in the chiller water over time, which inhibits the efficacy of FC. This phenomenon is supported in the literature [11]. It is also noted that as the day progresses the bacteria counts on chilled poultry begin to level off becoming more or less constant when leaving the tank. This seems to provide strong evidence justifying the assumption of our previous work that the system settles into an equilibrium if given enough time [10]. The advantage of the present model (10) is that its results do not rely on an equilibrium assumption.

In Fig. 3, we directly compare the stochastic and deterministic simulations (in the context of $25 \mathrm{mg} / \mathrm{L} \mathrm{FC}$ input). Recall that the deterministic initial bacteria level for every carcass was chosen to be the average bacteria count from the stochastic simulation. Thus, even though the variation in the initial counts for the stochastic simulation is large, through the chilling process these values converge to a neighborhood around the deterministic value. Generally speaking, this predicted behavior provides validation for our model (10) (and our wellmixing assumption) as this phenomenon is reflected in the literature. For instance, several experiments involving commercial chilling operations report, in general, a decrease in the standard deviation of Campylobacter levels from pre to post-chill carcasses $[5,7,23]$.

In connection with this typical "regularization" effect on carcass pathogen levels during chilling, model (10) can quantify the efficacy of FC control. We consider model (10) outputs when the input chlorine concentration is varied. This is governed by the parameter $c_{1}$ in the model. We can see in Fig. 4 that the FC level has a significant impact on the kill rate of the bacteria, but that relative effectiveness wanes as $c_{1}$ increases. Furthermore, running stochastic simulations we see that for incoming uniformly distributed Campylobacter loads on poultry with mean $2.16 \times 10^{4} \mathrm{CFU}$ and standard deviation (std dev) $1.03 \times 10^{4}$ $\mathrm{CFU}$, when $c_{1}=0$ (i.e. no FC input, see red curve in Fig. 4 (right)), the post-chill distribution has mean $2 \times 10^{4} \mathrm{CFU}$ and std dev $8.47 \times 10^{3}$ CFU. For $c_{1}=25$ (i.e. $25 \mathrm{mg} / \mathrm{L}$ input, see blue curve in Fig. 4 (right)), the post-chill distribution has mean $3.62 \times 10^{3} \mathrm{CFU}$ and std dev $8.55 \times 10^{2}$ CFU. Finally, for $c_{1}=50$, (see the green curve in Fig. 4 (right)), the post-chill distribution has mean $2.41 \times 10^{3} \mathrm{CFU}$ and std $\operatorname{dev} 1.69 \times 10^{3} \mathrm{CFU}$. This illustrates that FC input not only decreases Campylobacter levels on chilled poultry on average but can reduce the variation of post-chill pathogen levels on carcasses.
The results illustrated in Fig. 4 also have significant implications for cross-contamination dynamics during chilling. For instance, for chilling operations using no FC input, cross-contamination is more likely during the early stages of a processing shift. Referring to Fig. 4 (the red curves in both sub-Figures), we see that pathogen levels start relatively high on chilled carcasses but these levels decrease as processing continues. The reason for this concerns the competing dynamics of pathogen shed and attachment (set $C=0$ in Eq. (4)) as the carcass moves through the tank. Note that $W$ (pathogen level in the water) is near its max during this initial phase (see the dark blue curve in Fig. 5), and since there is not too much organic material in the tank to "pick" up the pathogens in the water (see Fig. 6 (left)), the attachment dynamic (which directly depends on the magnitude of $W$ ) is quite strong as compared with the shed dynamic. In stark contrast to this, for moderate to high concentrations of FC input, bacteria levels in the water are quite low during the early stage of chilling as the water is relatively free from organics. In this case, as the organic load builds, FC levels drop and pathogen levels in the water increase, magnifying the likelihood of cross-contamination later in the chilling day. This accounts for the increase of pathogen levels on post-chill carcasses as chilling progresses; see Fig. 4 (the blue and green curves in both sub-Figures).

The continued increase of the organic load in the water during chilling also provides justification for the model (10) predictions of FC dynamics in the chiller water. In Fig. 5 (left), we track the FC level in time. At the start of the day there is an initial spike in chlorine corresponding to FC addition into relatively "clean" water. However, as chicken byproducts/organic material build up in the tank, FC begins to be neutralized, corresponding to a decrease in FC values. Finally, FC levels begin to equilibrate as the addition of FC and removal rates of the organic material (due to overflow) in the chiller tank balance in time. This also justifies the equilibrium assumption in our previous averaged model [10].

In addition, Fig. 5 (right) displays the sensitivity of $W$ to stochasticity which logically follows the fact that the dynamics $W$ and $v_{P_{i}}$ are intimately tied together. What is interesting here is that the effects of stochasticity on $W$ are more significant for little or no FC input as opposed to high FC input levels. Given that cross-contamination risk is correlated to the magnitude of $W$, this suggests that stochastic dynamics are important to include in the context of evaluating processing operations where little or no FC input is used. On the other hand, for processes utilizing the upper limits of FC input, an averaged model may be sufficient [10]. Also, consistent with experiments using FC input is the idea that the bacteria level in the water is only marginally larger than the bacteria count on a single chicken $[4,8]$.

In, Fig. 6 (left), we track the dynamics of the organic load in the water as a function of time. The simulations show that this quantity is independent of the stochasticity and input chlorine levels. It also appears to be leveling off as time progresses. Finally, in Fig. 6 (right) we also track the dynamics of the organic load remaining on each chicken versus time. A similar trajectory is observed here as in the bacteria counts $v_{p i}$. As before, the stochastic levels converge to a variance window around the deterministic solution.

\subsection{Validation against experimental data}

The model (10) developed herein can be used for a wide variety of chilling scenarios. In particular, the results presented in this section are framed in the context of the experiments in [7]. In this work the authors demonstrate a log decrease from the start $\left[10^{4}, 10^{4.6}\right]$ to end of the chilling process $\left[10^{31}, 10^{3.9}\right]$. We apply the same experimental settings used when choosing the parameters (e.g., chlorine input levels $c_{0}=35 \mathrm{mg} / \mathrm{L}$, initial counts $J_{p, 3}=0.02 \mathrm{~kg}$ or $1 \%$ of mass). Using the same initial distribution of bacteria counts (see Fig. 3), the model recovers the same dynamics in bacterial counts and the approximately 1 log decrease in the final pathogen levels. For additional testing, the model was compared to an experimental setup involving multiple tanks 

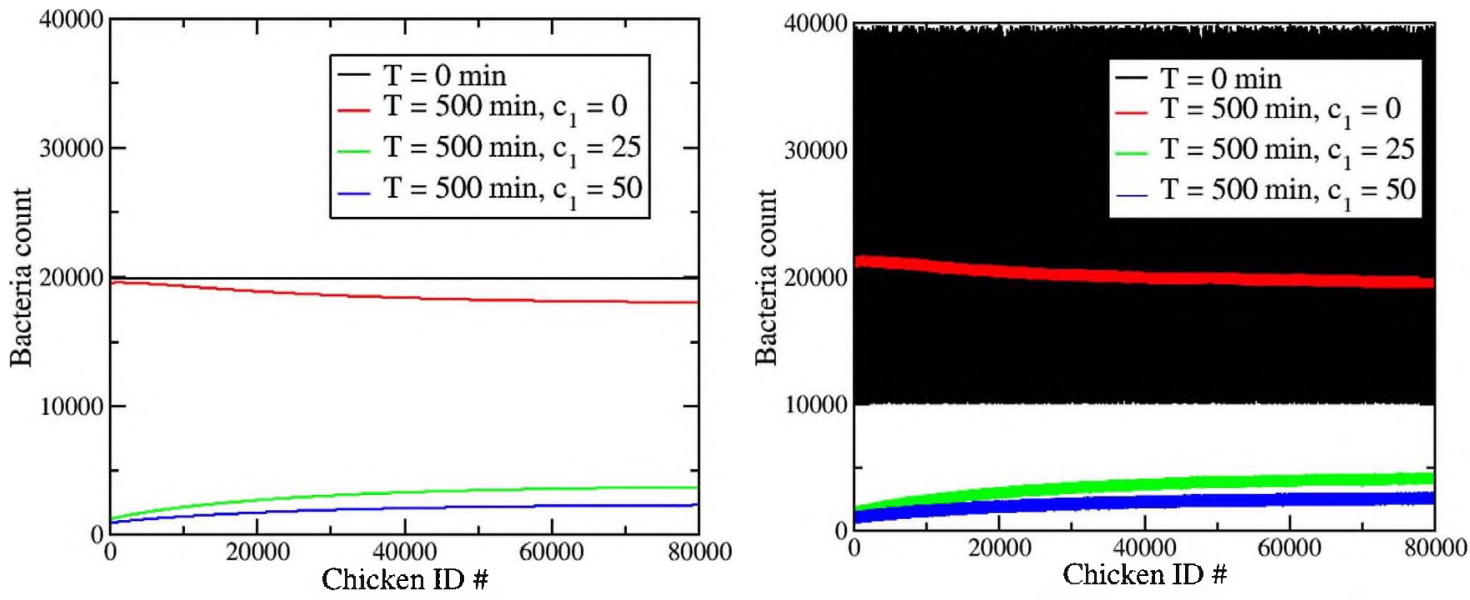

Fig. 4. Starting and final Campylobacter count distributions as a function of the injection chlorine level. Left: Deterministic and Right: Stochastic.
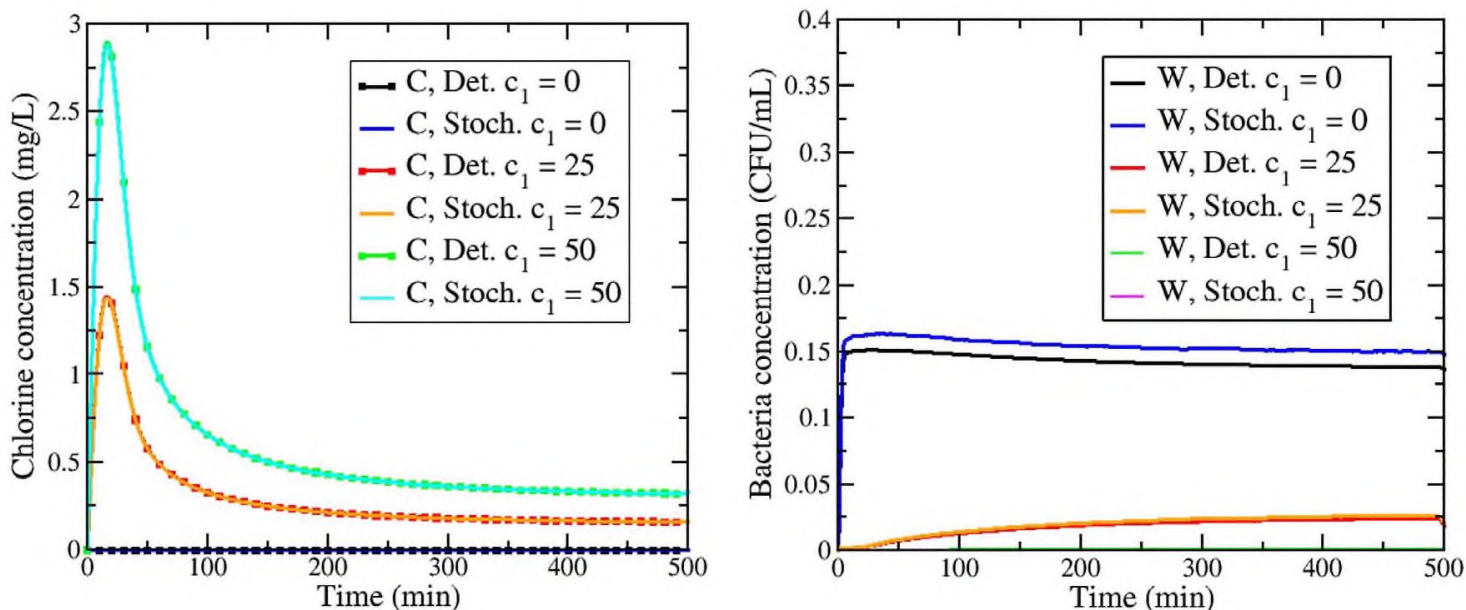

Fig. 5. Left: chlorine versus time for the different input concentrations. Right dynamics of Campylobacter levels in the water in units of concentration of bacteria per milliliter.
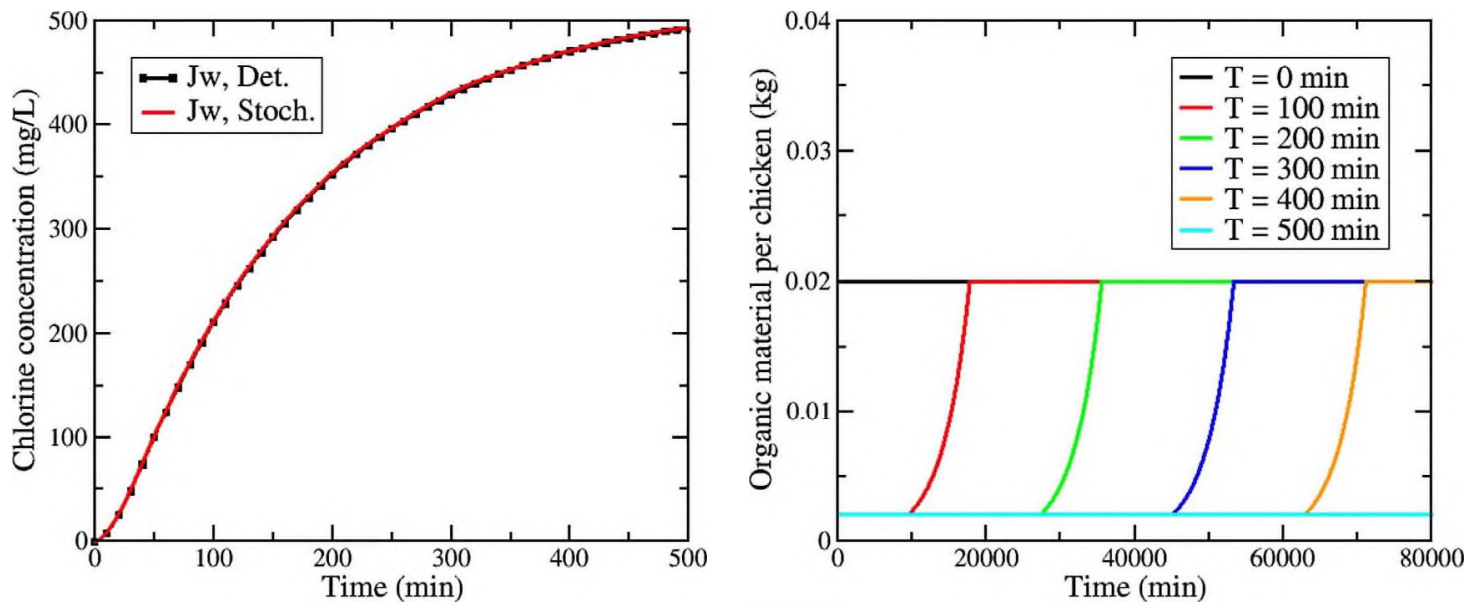

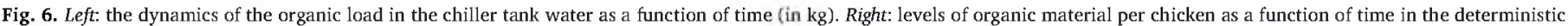
run. FC input is $25 \mathrm{mg} / \mathrm{L}$.

with recirculation/filtration of the chiller water. The initial counts, $J_{p .0}=0.02 \mathrm{~kg}$, and chlorine input levels, $c_{0}=30 \mathrm{mg} / \mathrm{L}$, were chosen to match the experimental setup of [4]. In that work, the observed decrease was approximately 2 logs; whereas, the model presented here still predicts a 1-1.5 log decrease. This difference can be accounted for by the fact that in [4] their experimental process allows for re-circulation of water, which is not present in the model. This re-circulation would allow for less bacteria in the chill water leading to lower bacteria counts in the presence of the same chlorine levels. In comparison to these two recent experimental works, model (10) shows good quantitative agreement, suggesting that the model captures the main mechanisms involved. While more specified experimental data could be used to refine the model further, given the results above, the model has proven useful as a predictive tool for tracking contamination during 


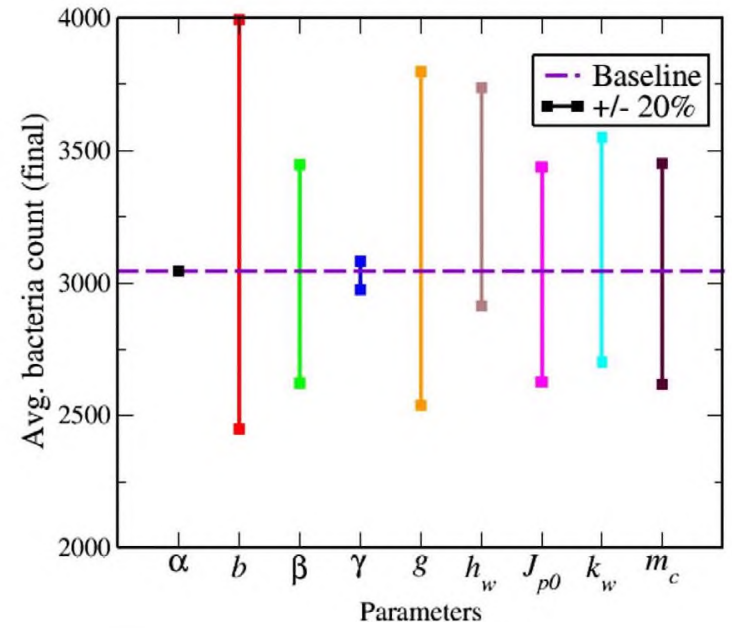

Fig. 7. Sensitivity analysis. Each parameter's value is increased or decreased by $20 \%$ from its baseline value in Table 1 . The average final counts on carcasses leaving the chiller tank are compared. Larger ranges correspond to more sensitive parameters.

chilling. In addition, the strength of the model is its adaptability to cover a wide range of scenarios involving different dwell times, chlorine levels, rate of poultry entry and initial bacteria counts. They key to the model's reliability is the accurate selection of parameters for a given experimental setup. We probe this question in the next section with a sensitivity analysis of the model parameters to indicate their relative importance in determining final pathogen levels on the carcasses.

\subsection{Sensitivity analysis}

In this section, we test the relative sensitivity of each of the main parameters in Table 1 on the final pathogen levels. This analysis is conducted in a systematic way using the baseline values from Section 5 . We isolate the effect of each parameter by running simulations in which the given parameter is increased or decreased by $20 \%$, while the other parameters are fixed at baseline. We compare the results of the average bacteria level per carcass at the end of the process day. Changing parameters relative to their magnitude allows for direct comparison of relative strengths. The results of this sensitivity analysis are summarized in Fig. 7 where the larger range of final counts corresponds to a more sensitive parameter. Our results indicate that the model is most sensitive to $b$ the shed rate of bacteria and $g$ the refresh rate of water per carcass processed. The model is least sensitive to $\alpha$ (the scaling parameter that relates the pathogen kill rate in the water to the kill rate on carcass surfaces) and $\gamma$ (the shed rate of organic matter from the carcass into the tank). This suggests that the model is most effective when the shed rate of bacteria is well estimated and the inflow rate of chlorinated water is well-controlled. In order to use the model (10) to gain insight into management strategies, especially concerning intra and inter-flock contamination, that are built on the interplay between chiller water chemistry dynamics, timing within a processing shift, and the microbial distribution among pre-chill carcasses, commercial scale experiments should be conducted to better estimate the shed parameter $b$.

\section{Conclusions}

Although cross-contamination during immersion chilling involves a complex interaction of water chemistry and microbial dynamics as well as industrial specifications, our IBM (10) is able to mathematically elucidate these relationships in a manner that is useful for risk assessment. For instance, referring to Section 5 results, using parameters determined by the experimental setup in [7], we were able to quantify the pathogen load decrease during a typical $8 \mathrm{~h}$ chilling shift. In the context of QMRA models (see [1] for current examples), once parameter values associated to the particular processing conditions are established, model (10) can be used as a tool to quantify post-chilling pathogen loads on broiler carcasses. The significance here is that the IBM (10) is capable of taking input drawn from distributions of microbial levels on pre-chiller carcasses and subsequently providing an output distribution of microbial contamination on carcasses exiting the chiller tank at any time point during a typical processing shift. Notice that $(10)$ is also easily amenable to comply with various specifications involved with immersion chilling. In addition, while the IBM (10) can address more delicate questions involving both the prevalence and concentration of pathogens on carcasses during chilling, it also provides justification for when our equilibrium approach [10] may be sufficient (for instance, for analyzing pathogen levels on carcasses towards the end of the chiller processing day).

Concerning future directions, a key question to explore is whether the variance in outputs from stochastic solutions (see Fig. 3 , for instance) can be predicted in terms of the model parameters and information characterizing input distributions. We believe this may be possible in the case of a uniform input distribution (as presented here for incoming microbial load and organic material on carcasses), but could be quite complicated if sampling from a different distribution. In addition, referring to Fig. 4, one could do a cost-benefit analysis to determine if doubling the chlorine concentration from 25 to $50 \mathrm{mg} / \mathrm{L}$ is worth the added cost. Finally, we note that stochasticity could be implemented in other parameters.

\section{Acknowledgements}

This work was supported by a research contract between the Public Health Agency of Canada, York University and Cleveland State University. Jianhong $\mathrm{Wu}$ is also supported by the Natural Sciences and Engineering Research Council of Canada (Grant number: 1055882011) and the Canada Research Chairs Program (Grant number: 230720). The authors want to thank the CFIA for their support and details concerning poultry slaughter establishments which operate under the CFIA approved Modernized Poultry Inspection Program. The authors also wish to thank the two anonymous referees for their careful reading and suggestions to improve the manuscript.

\section{References}

[1] B. Chapman, A. Otten, A. Fazil, N. Emst, B. Smith, A review of quantitative microbial risk assessment and consumer process models for Campylobacter in broiler chickens, Microb. Risk Anal. 2 (2016) 3-15

[2] M. Nauta, A. HIll, H. Rosenquist, S. Brynestad, A. Fetsch, P. van der Logt, A. Fazil, B. Christensen, E. Katsma, B. Borck, A. Havelaar, A comparison of risk assessments on Campylobacter in broiler meat, Int. J. Food Microbiol. 129 (2009) 107-123.

[3] C.J. Thomas, T.A. McMeekin, Contamination of broiler carcass skin during commercial processing procedures: an electron microscopic study, Appl. Environ. Microbiol. 40 (1980) 133-144.

[4] J.K. Northcutt. D. Smith, R. I. Huezo, K.D. Ingram, Microbiology of broiler carcasses and chemistry of chiller water as affected by water reuse, Poult. Sci. 87 (2008) 1458-1463.

[5] T. Seliwiorstow, J. Bar, I.V. Damme, M. Uyttendaele, L.D. Zutter, Campylobacter Carcass contamination throughout the slaughter process of Campylobacter-positive broiler batches, Int. J. Food Microbiol. 194 (2015) 25-31.

[6] H. Yang, Y. Li, M.G. Johnson, Survival and death of Salmonella Typhimurium and Campylobacter jejuni in processing water and on chicken skin during poultry scalding and chilling, J. Food Prot. 64 (2001) 770-776.

[7] M.E. Berrang, J.A. Dickens, Presence and level of Campylobacter spp. on broiler carcasses throughout the processing plant, J. Appl. Poult. Res. 9 (2000) 43-47.

[8] J.K. Northcutt, J.A. Gason. D.P. Smith. R.I. Buhr. D.L. Fletchert, Broiler carcass bacterial counts after immersion chilling using either a low or high volume of water, Poult. Sci. 85 (2006) 1802-1806.

[9] O. Bucher, A.M. Farrar, S.C. Totton, W. Wilkins, L.A. Waddell, B.J. Wihelm, S.A. McEwen, A. Fazil, A. Rajic, A systematic review-meta-analysis of chilling in terventions and a meta-regression of various processing interventions for Salmonella contamination of chicken, Prev. Vet. Med. 103 (2012) 1-15.

[10] D. Munther, X. Sun, Y. Xiao, S. Tang, H. Shimozako, J. Wu. B. Smith, A. Fazil, Modeling cross-contamination during poultry processing: dynamics in the chiller tank, Food Control 59 (2016) 271-281.

[11] S.M. Russell, Controlling Salmonella in Poultry Production and Processing, CRC 
Press, Boca Raton, FL, 2012.

[12] K. Jang, M. Kim, S. Ha, K. Kim, K. Lee, D. Chung, G. Kim, K. Kim, Morphology and adhesion of Campylobacter jejuni to chicken skin under varying conditions, J. Microbiol. Biotechnol. 17 (2007) 202-206.

[13] D.E. Helbling, J.M. VanBriesen, Free chlorine demand and cell survival of microbial suspensions, Water Res. 41 (2007) 4424-4434

[14] B. Zhang, Y. Luo, B. Zhou, Q. Wang, P. D. Millner, A novel microfluidic mixer-based approach for determining inactivation kinetics of Escherichia coli 0157:H7 in chlorine solutions, Food Microbiol. 49 (2015) 152-160.

[15] D.A. Ratkowsky, J. Olley, T.A. McMeekin, A. Ball, Relationship between tempera ture and growth rate of bacterial cultures, J. Bacteriol. 149 (1982) 1-5.

[16] G. Wang, M.P. Doyle, Survival of enterohemorrhagic Escherichia coli O157:H7 in water, J. Food Prot. 6 (1998) 657-775.

[17] B.A.M. Carciofi, I.B. Laurindo, Water uptake by poultry carcasses during cooling by water immersion, Chem. Eng. Process. 46 (2007) 444-450.

[18] A.F. Filipov, Differential Equations with Discontinunus Righthand Sides, Springer-
Science + Business Media, Dordrecht, 1988.

[19] USDA, Fsis directive, veritying sanitary dressing and process control procedures by off-line ipp in poultry slaughterhouse operations, effective October 28, 2012, 2012 (http://www.fsis. usda.gov). Accessed: January 2014.

[20] M.J. Blaser, P.F. Smith, W.L. Wang, J.C. Hoff, Inactivation of Campylobacter jejuni by chlorine and monochloramine, Appl. Environ. Microbiol. 51 (1986) 307-311.

[21] L.S. Tsai, J.E. Schade, B.T. Molyneux, Chlorination of poultry chiller water: chlorine demand and disinfection efficiency, Poult. Sci. 71 (1992) 188-196.

[22] R. Cavani, R.P. Schocken-Iturrino, T.C.F.L. Garcia, A.C. de Oliveira, Comparison of microbial load in immersion chilling water and poultry carcasses after 8,16 and 24 working hours, Ciencia Rural 40 (2010) 1603-1609.

[23] M. Kameyama, T. Chuma, T. Nishimoto, H. Oniki, Y. Yanagitani, R. Kanetou, K. Gotou, F. Shahada, H. Iwata, K. Okamoto, Effect of cooled and chlorinated chiller water on Campylobacter and coliform counts on broiler carcasses during chilling at a middle-size poultry processing plant, J. Veter. Med. Sc. Jpnese Soc. Veter. Sci. 74 (2012) 129-133. 